Acta Crystallographica Section E

Structure Reports

Online

ISSN 1600-5368

\section{1-Isopropyl-4-tosylpiperazin-1-ium trifluoroacetate}

\section{Jiang-Sheng $\mathrm{Li}^{\mathrm{a}}{ }^{\mathrm{*}}$ Dao-Wu Yang ${ }^{\mathrm{a}}$ and Wei-Dong Liu ${ }^{\mathrm{b}}$}

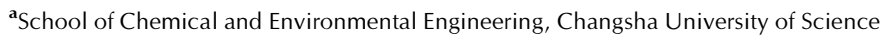
and Technology, Changsha 410076, People's Republic of China, and ${ }^{\mathbf{b}}$ Hunan Research Institute of Chemical Industry, Changsha 410007, People's Republic of China

Correspondence e-mail: jansenlee1103@yahoo.com.cn

Received 20 November 2007; accepted 21 November 2007

Key indicators: single-crystal X-ray study; $T=113 \mathrm{~K}$; mean $\sigma(\mathrm{C}-\mathrm{C})=0.004 \AA$; disorder in solvent or counterion; $R$ factor $=0.054 ; w R$ factor $=0.124$; data-toparameter ratio $=11.9$.

In the title compound, $\mathrm{C}_{14} \mathrm{H}_{23} \mathrm{~N}_{2} \mathrm{O}_{2} \mathrm{~S}^{+} \cdot \mathrm{C}_{2} \mathrm{~F}_{3} \mathrm{O}_{2}{ }^{-}$, the piperazine ring adopts a chair conformation. The crystal packing is stabilized by $\mathrm{C}-\mathrm{H} \cdots \mathrm{O}$ and $\mathrm{N}-\mathrm{H} \cdots \mathrm{O}$ hydrogen bonds between the cation and anion. The $\mathrm{F}$ atoms are disordered over two positions; the site occupancy factors are 0.55 (2) and 0.45 (2).

\section{Related literature}

For related literature on benzenesulfonamides, see: Yu et al. (2007); Xing et al. (2006, 2005).<smiles>Cc1ccc(S(=O)(=O)N2CC[NH+](C(C)C)CC2)cc1</smiles>

\section{Experimental}

\section{Crystal data}

$\mathrm{C}_{14} \mathrm{H}_{23} \mathrm{~N}_{2} \mathrm{O}_{2} \mathrm{~S}^{+} \cdot \mathrm{C}_{2} \mathrm{~F}_{3} \mathrm{O}_{2}^{-}$

$M_{r}=396.42$

Monoclinic, $P 2_{1} / n$

$$
\begin{aligned}
& \beta=105.87(3)^{\circ} \\
& V=1833.8(6) \AA^{3} \\
& Z=4 \\
& \text { Mo } K \alpha \text { radiation }
\end{aligned}
$$

$\mu=0.23 \mathrm{~mm}^{-1}$

$T=113(2) \mathrm{K}$

$0.10 \times 0.08 \times 0.02 \mathrm{~mm}$

\section{Data collection}

Rigaku Saturn diffractometer

Absorption correction: multi-scan

(CrystalClear;

Rigaku/MSC, 2005)

$T_{\min }=0.977, T_{\max }=0.995$

Refinement

$R\left[F^{2}>2 \sigma\left(F^{2}\right)\right]=0.054$

$w R\left(F^{2}\right)=0.124$

$S=1.03$

3226 reflections

270 parameters

43 restraints

10902 measured reflections 3226 independent reflections 2517 reflections with $I>2 \sigma(I)$ $R_{\text {int }}=0.072$

$\mathrm{H}$ atoms treated by a mixture of independent and constrained refinement

$\Delta \rho_{\max }=0.40{\mathrm{e} \AA^{-3}}^{-3}$

$\Delta \rho_{\min }=-0.28 \mathrm{e} \AA^{-3}$

Table 1

Hydrogen-bond geometry $\left(\AA{ }^{\circ}\right)$.

\begin{tabular}{lllll}
\hline$D-\mathrm{H} \cdots A$ & $D-\mathrm{H}$ & $\mathrm{H} \cdots A$ & $D \cdots A$ & $D-\mathrm{H} \cdots A$ \\
\hline $\mathrm{N} 2-\mathrm{H} 2 A \cdots \mathrm{O} 4^{\mathrm{i}}$ & $0.902(11)$ & $1.828(12)$ & $2.724(3)$ & $172(3)$ \\
$\mathrm{C} 9-\mathrm{H} 9 A \cdots \mathrm{O} 2^{\mathrm{ii}}$ & 0.97 & 2.52 & $3.405(4)$ & 151 \\
$\mathrm{C} 10-\mathrm{H} 10 A \cdots \mathrm{O} 4^{\mathrm{ii}}$ & 0.97 & 2.45 & $3.357(4)$ & 155 \\
$\mathrm{C} 2-\mathrm{H} 2 \cdots \mathrm{O} 3^{\text {iii }}$ & 0.93 & 2.58 & $3.225(4)$ & 127 \\
\hline
\end{tabular}

Symmetry codes: (i) $x+1, y, z$; (ii) $-x+1,-y,-z$; (iii) $x+1, y-1, z$.

Data collection: CrystalClear (Rigaku/MSC, 2005); cell refinement: CrystalClear; data reduction: CrystalClear; program(s) used to solve structure: SHELXS97 (Sheldrick, 1997); program(s) used to refine structure: SHELXL97 (Sheldrick, 1997); molecular graphics: SHELXTL (Bruker, 1997); software used to prepare material for publication: CrystalStructure (Rigaku/MSC, 2005).

Supplementary data and figures for this paper are available from the IUCr electronic archives (Reference: BT2629).

\title{
References
}

Bruker (1997). SHELXTL. Bruker AXS Inc., Madison, Wisconsin, USA.

Rigaku/MSC (2005). CrystalClear and CrystalStructure. Rigaku Corporation, Tokyo, Japan.

Sheldrick, G. M. (1997). SHELXS97 and SHELXL97. University of Göttingen, Germany.

Xing, J.-D., Bai, G.-Y., Zeng, T. \& Li, J.-S. (2006). Acta Cryst. E62, o79-o80.

Xing, J.-D. \& Zeng, T. (2005). Acta Cryst. E61, o4318-o4319.

Yu, H.-J. \& Li, J.-S. (2007). Acta Cryst. E63, o3766. 


\section{supporting information}

Acta Cryst. (2008). E64, o22 [https://doi.org/10.1107/S1600536807061570]

\section{1-Isopropyl-4-tosylpiperazin-1-ium trifluoroacetate}

\section{Jiang-Sheng Li, Dao-Wu Yang and Wei-Dong Liu}

\section{S1. Comment}

In the title compound (Fig. 1) both $\mathrm{N}$ atoms have a pyramidal arrangement, but the pyramid of the amide is somewhat shallower than that of the protonated $\mathrm{N}$. The protonated piperazin ring adopts a chair conformation.

The crystal packing is stabilized by $\mathrm{C}-\mathrm{H} \cdots \mathrm{O}$ and $\mathrm{N}-\mathrm{H} \cdots \mathrm{O}$ hydrogen bonds (Table 1) between the cation and anion. Weak intermolecular $\mathrm{C}-\mathrm{H} \cdots \mathrm{O}$ hydrogen bonds involving an $\mathrm{S}=\mathrm{O}$ group as acceptor play an important role in the moleclular packing.

\section{S2. Experimental}

A solution of 4-methylbenzenesulfonyl chloride $(3.28 \mathrm{~g}, 17 \mathrm{mmol})$ in $\mathrm{CH}_{2} \mathrm{Cl}_{2}$ was added dropwise to a mixture of 1-isopropylpiperazine $(2.23 \mathrm{~g}, 82 \%, 0.14 \mathrm{mmol})$ and sodium biscarbonate $(3.36 \mathrm{~g}, 40 \mathrm{mmol})$ in $\mathrm{CH}_{2} \mathrm{Cl}_{2}(30 \mathrm{ml})$ at room temperature with stirring. After stirring for $4 \mathrm{~h}$ followed by filtration, the organic filtrate was rotoevaporated under vacuum. The resulting solid, in a yield of $53.8 \%$, was purified by recrystallization from methanol. Crystals in the form of colourless blocks were grown by evaporation of a trifluoroacetic solution.

\section{S3. Refinement}

The N-bound $\mathrm{H}$ atoms were refined freely with the restraint of 0.90 (1) $\AA$, while the other $\mathrm{H}$ atoms were positioned geometrically $(\mathrm{C}-\mathrm{H}=0.93,0.96$ and $0.97 \AA)$ and refined as riding with $U_{\text {iso }}(\mathrm{H})=1.2 U_{\text {eq }}(\mathrm{C})$ or $1.5 U_{\text {eq }}($ methyl C). The three $\mathrm{F}$ atoms are disordered over two site occupancy of 0.55 (2): 0.45 (2). The $\mathrm{C}-\mathrm{F}$ distances were restrained to 1.36 (1) $\AA$ and their displacement parameters were restrained to be isotropic by means of the instruction ISOR (tolerance $0.01)$ in SHELXL. 

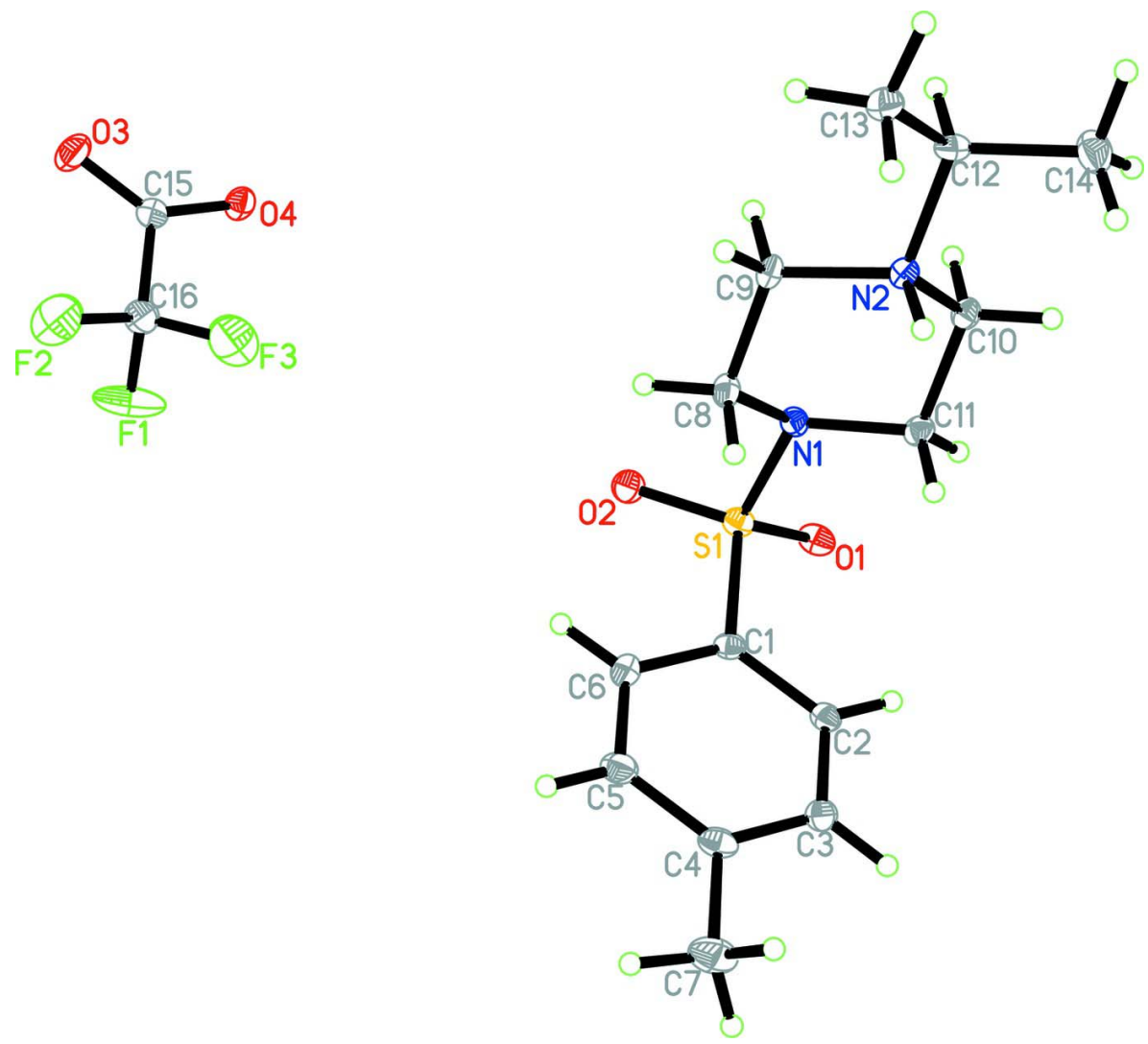

Figure 1

The molecular structure of (I) with the atom-numbering scheme and 30\% probability displacement ellipsoids. Only one component of the disordered $\mathrm{CF}_{3}$ group is shown.

1-Isopropyl-4-tosylpiperazin-1-ium trifluoroacetate

Crystal data

$\mathrm{C}_{14} \mathrm{H}_{23} \mathrm{~N}_{2} \mathrm{O}_{2} \mathrm{~S}^{+} \cdot \mathrm{C}_{2} \mathrm{~F}_{3} \mathrm{O}_{2}^{-}$

$M_{r}=396.42$

Monoclinic, $P 2_{1} / n$

Hall symbol: -P $2 \mathrm{yn}$

$a=11.659(2) \AA$

$b=8.4274$ (17) $\AA$

$c=19.404(4) \AA$

$\beta=105.87(3)^{\circ}$

$V=1833.8(6) \AA^{3}$

$Z=4$

$F(000)=832$

$D_{\mathrm{x}}=1.436 \mathrm{Mg} \mathrm{m}^{-3}$

Mo $K \alpha$ radiation, $\lambda=0.71073 \AA$

Cell parameters from 3880 reflections

$\theta=2.2-27.9^{\circ}$

$\mu=0.23 \mathrm{~mm}^{-1}$

$T=113 \mathrm{~K}$

Block, colorless

$0.10 \times 0.08 \times 0.02 \mathrm{~mm}$

\section{Data collection}

Rigaku Saturn

diffractometer

Radiation source: rotating anode

Confocal monochromator

$\omega$ scans

Absorption correction: multi-scan

(CrystalClear; Rigaku/MSC, 2005)

$T_{\min }=0.977, T_{\max }=0.995$
10902 measured reflections

3226 independent reflections

2517 reflections with $I>2 \sigma(I)$

$R_{\text {int }}=0.072$

$\theta_{\text {max }}=25.0^{\circ}, \theta_{\text {min }}=1.9^{\circ}$

$h=-13 \rightarrow 9$

$k=-9 \rightarrow 10$

$l=-23 \rightarrow 23$ 


\section{Refinement}

Refinement on $F^{2}$

Least-squares matrix: full

$R\left[F^{2}>2 \sigma\left(F^{2}\right)\right]=0.054$

$w R\left(F^{2}\right)=0.124$

$S=1.03$

3226 reflections

270 parameters

43 restraints

Primary atom site location: structure-invariant direct methods
Secondary atom site location: difference Fourier map

Hydrogen site location: inferred from neighbouring sites

$\mathrm{H}$ atoms treated by a mixture of independent and constrained refinement

$w=1 /\left[\sigma^{2}\left(F_{\mathrm{o}}^{2}\right)+(0.0512 P)^{2}+0.9104 P\right]$ where $P=\left(F_{\mathrm{o}}^{2}+2 F_{\mathrm{c}}^{2}\right) / 3$

$(\Delta / \sigma)_{\max }=0.003$

$\Delta \rho_{\max }=0.40 \mathrm{e} \AA^{-3}$

$\Delta \rho_{\min }=-0.28$ e $\AA^{-3}$

\section{Special details}

Geometry. All e.s.d.'s (except the e.s.d. in the dihedral angle between two 1.s. planes) are estimated using the full covariance matrix. The cell e.s.d.'s are taken into account individually in the estimation of e.s.d.'s in distances, angles and torsion angles; correlations between e.s.d.'s in cell parameters are only used when they are defined by crystal symmetry. An approximate (isotropic) treatment of cell e.s.d.'s is used for estimating e.s.d.'s involving 1.s. planes.

Refinement. Refinement of $F^{2}$ against ALL reflections. The weighted $R$-factor $w R$ and goodness of fit $S$ are based on $F^{2}$, conventional $R$-factors $R$ are based on $F$, with $F$ set to zero for negative $F^{2}$. The threshold expression of $F^{2}>\sigma\left(F^{2}\right)$ is used only for calculating $R$-factors(gt) etc. and is not relevant to the choice of reflections for refinement. $R$-factors based on $F^{2}$ are statistically about twice as large as those based on $F$, and $R$ - factors based on ALL data will be even larger.

Fractional atomic coordinates and isotropic or equivalent isotropic displacement parameters $\left(\AA^{2}\right)$

\begin{tabular}{|c|c|c|c|c|c|}
\hline & $x$ & $y$ & $z$ & $U_{\text {iso }} * / U_{\text {eq }}$ & Occ. $(<1)$ \\
\hline $\mathrm{S} 1$ & $0.63629(6)$ & $-0.19035(8)$ & 0.09809 (4) & $0.0214(2)$ & \\
\hline $\mathrm{F} 1$ & $0.0952(9)$ & 0.0965 (13) & $0.2419(6)$ & $0.078(3)$ & $0.55(2)$ \\
\hline $\mathrm{F} 2$ & 0.1367 (11) & $0.3388(12)$ & $0.2544(5)$ & $0.077(3)$ & $0.55(2)$ \\
\hline F3 & $0.2151(6)$ & 0.1905 (18) & $0.1909(5)$ & $0.070(3)$ & $0.55(2)$ \\
\hline $\mathrm{F} 1^{\prime}$ & $0.0745(9)$ & $0.175(2)$ & $0.2595(5)$ & $0.083(4)$ & $0.45(2)$ \\
\hline $\mathrm{F} 2^{\prime}$ & $0.1771(11)$ & $0.3557(10)$ & $0.2275(9)$ & 0.077 (4) & $0.45(2)$ \\
\hline F3' & $0.1949(11)$ & $0.1212(15)$ & $0.2000(6)$ & $0.066(4)$ & $0.45(2)$ \\
\hline $\mathrm{O} 1$ & $0.63011(18)$ & $-0.3355(2)$ & $0.05863(10)$ & $0.0265(5)$ & \\
\hline $\mathrm{O} 2$ & $0.53262(17)$ & $-0.1320(2)$ & $0.11580(10)$ & $0.0275(5)$ & \\
\hline $\mathrm{O} 3$ & $-0.0548(2)$ & 0.3661 & $0.14312(12)$ & $0.0410(6)$ & \\
\hline $\mathrm{O} 4$ & $0.00768(17)$ & $0.1625(2)$ & $0.08810(10)$ & $0.0277(5)$ & \\
\hline N1 & $0.6753(2)$ & $-0.0517(3)$ & $0.04892(11)$ & $0.0197(5)$ & \\
\hline $\mathrm{N} 2$ & $0.7924(2)$ & 0.1936 & $-0.01082(12)$ & $0.0183(5)$ & \\
\hline $\mathrm{C} 1$ & $0.7539(2)$ & $-0.2013(3)$ & $0.17719(14)$ & $0.0195(6)$ & \\
\hline $\mathrm{C} 2$ & $0.8553(3)$ & $-0.2897(3)$ & $0.17916(14)$ & $0.0211(6)$ & \\
\hline $\mathrm{H} 2$ & 0.8588 & -0.3517 & 0.1402 & $0.025^{*}$ & \\
\hline $\mathrm{C} 3$ & $0.9508(3)$ & $-0.2842(4)$ & $0.23978(15)$ & $0.0254(7)$ & \\
\hline $\mathrm{H} 3$ & 1.0186 & -0.3440 & 0.2415 & $0.030 *$ & \\
\hline $\mathrm{C} 4$ & $0.9472(3)$ & -0.1907 (3) & $0.29824(14)$ & $0.0248(7)$ & \\
\hline $\mathrm{C} 5$ & $0.8443(3)$ & -0.1060 & $0.29589(15)$ & $0.0281(7)$ & \\
\hline H5 & 0.8406 & -0.0447 & 0.3351 & $0.034^{*}$ & \\
\hline C6 & $0.7468(3)$ & $-0.1113(3)$ & $0.23611(15)$ & $0.0258(7)$ & \\
\hline H6 & 0.6776 & -0.0556 & 0.2353 & $0.031^{*}$ & \\
\hline $\mathrm{C} 7$ & $1.0535(3)$ & -0.1813 & $0.36274(16)$ & $0.0373(8)$ & \\
\hline
\end{tabular}




$\begin{array}{lllll}\text { H7A } & 1.0736 & -0.2858 & 0.3820 & 0.056^{*} \\ \text { H7B } & 1.0348 & -0.1150 & 0.3984 & 0.056^{*} \\ \text { H7C } & 1.1199 & -0.1371 & 0.3490 & 0.056^{*} \\ \text { C8 } & 0.6947(3) & 0.1074(3) & 0.08214(14) & 0.0222(6) \\ \text { H8A } & 0.7690 & 0.1081 & 0.1200 & 0.027^{*} \\ \text { H8B } & 0.6305 & 0.1321 & 0.1033 & 0.027^{*} \\ \text { C9 } & 0.6995(3) & 0.2314(3) & 0.02688(14) & 0.0215(7) \\ \text { H9A } & 0.6221 & 0.2383 & -0.0080 & 0.026^{*} \\ \text { H9B } & 0.7169 & 0.3339 & 0.0501 & 0.026^{*} \\ \text { C10 } & 0.7722(2) & 0.0296(3) & -0.04174(14) & 0.0201(6) \\ \text { H10A } & 0.8350 & 0.0033 & -0.0639 & 0.024^{*} \\ \text { H10B } & 0.6967 & 0.0261 & -0.0785 & 0.024^{*} \\ \text { C11 } & 0.7712(3) & -0.0907(3) & 0.01589(14) & 0.0213(6) \\ \text { H11A } & 0.7585 & -0.1962 & -0.0049 & 0.026^{*} \\ \text { H11B } & 0.8474 & -0.0898 & 0.0520 & 0.026^{*} \\ \text { C12 } & 0.7963(3) & 0.3177(3) & -0.06701(14) & 0.0221(6) \\ \text { H12 } & 0.7191 & 0.3189 & -0.1033 & 0.026^{*} \\ \text { C13 } & 0.8190(3) & 0.4804(3) & -0.03294(16) & 0.0271(7) \\ \text { H13A } & 0.8896 & 0.4772 & 0.0066 & 0.041^{*} \\ \text { H13B } & 0.7522 & 0.5112 & -0.0161 & 0.041^{*} \\ \text { H13C } & 0.8296 & 0.5558 & -0.0677 & 0.041^{*} \\ \text { C14 } & 0.8927(3) & 0.2765(4) & -0.10319(17) & 0.0337(8) \\ \text { H14A } & 0.8996 & 0.3606 & -0.1352 & 0.051^{*} \\ \text { H14B } & 0.8720 & 0.1797 & -0.1298 & 0.051^{*} \\ \text { H14C } & 0.9675 & 0.2631 & -0.0675 & 0.051^{*} \\ \text { C15 } & 0.0119(3) & 0.2552(3) & 0.13959(15) & 0.0241(7) \\ \text { C16 } & 0.1144(3) & 0.2243(4) & 0.20678(16) & 0.0313(8) \\ \text { H2A } & 0.8653(14) & 0.193(4) & 0.0208(13) & 0.031(9)^{*} \\ & & & & \end{array}$

Atomic displacement parameters $\left(\AA^{2}\right)$

\begin{tabular}{lllllll}
\hline & $U^{11}$ & $U^{22}$ & $U^{33}$ & $U^{12}$ & $U^{13}$ & $U^{23}$ \\
\hline S1 & $0.0190(4)$ & $0.0236(4)$ & $0.0199(4)$ & $-0.0031(3)$ & $0.0026(3)$ & $0.0009(3)$ \\
F1 & $0.079(5)$ & $0.074(5)$ & $0.064(4)$ & $-0.015(3)$ & $-0.010(3)$ & $0.047(4)$ \\
F2 & $0.086(5)$ & $0.073(5)$ & $0.046(4)$ & $0.026(4)$ & $-0.027(3)$ & $-0.032(3)$ \\
F3 & $0.028(3)$ & $0.129(7)$ & $0.050(3)$ & $0.014(4)$ & $0.005(2)$ & $0.008(5)$ \\
F1 $^{\prime}$ & $0.077(5)$ & $0.139(9)$ & $0.036(4)$ & $0.013(6)$ & $0.019(3)$ & $0.036(5)$ \\
F2 $^{\prime}$ & $0.063(5)$ & $0.046(4)$ & $0.085(7)$ & $-0.023(3)$ & $-0.040(4)$ & $-0.008(4)$ \\
F3' & $0.062(6)$ & $0.063(6)$ & $0.052(5)$ & $0.044(4)$ & $-0.021(4)$ & $-0.016(4)$ \\
O1 & $0.0317(13)$ & $0.0237(11)$ & $0.0212(10)$ & $-0.0075(9)$ & $0.0025(9)$ & $-0.0013(8)$ \\
O2 & $0.0180(12)$ & $0.0362(13)$ & $0.0290(11)$ & $0.0000(9)$ & $0.0075(9)$ & $0.0045(9)$ \\
O3 & $0.0448(15)$ & $0.0332(13)$ & $0.0382(13)$ & $0.0159(11)$ & $0.0001(11)$ & $-0.0083(11)$ \\
O4 & $0.0216(12)$ & $0.0312(12)$ & $0.0270(11)$ & $0.0039(9)$ & $0.0012(9)$ & $-0.0056(9)$ \\
N1 & $0.0213(14)$ & $0.0194(13)$ & $0.0194(12)$ & $0.0001(10)$ & $0.0074(10)$ & $0.0004(10)$ \\
N2 & $0.0172(13)$ & $0.0179(12)$ & $0.0191(12)$ & $0.0001(10)$ & $0.0037(10)$ & $-0.0019(10)$ \\
C1 & $0.0223(16)$ & $0.0160(14)$ & $0.0187(14)$ & $-0.0008(11)$ & $0.0031(12)$ & $0.0019(11)$ \\
C2 & $0.0250(17)$ & $0.0199(15)$ & $0.0186(14)$ & $-0.0006(12)$ & $0.0064(12)$ & $0.0019(12)$ \\
C3 & $0.0217(17)$ & $0.0302(17)$ & $0.0258(15)$ & $0.0025(13)$ & $0.0091(13)$ & $0.0056(13)$
\end{tabular}


supporting information

\begin{tabular}{lllllll} 
C4 & $0.0262(18)$ & $0.0242(16)$ & $0.0214(15)$ & $-0.0057(13)$ & $0.0021(13)$ & $0.0049(12)$ \\
C5 & $0.037(2)$ & $0.0244(17)$ & $0.0201(15)$ & $0.0035(13)$ & $0.0032(14)$ & $0.0000(12)$ \\
C6 & $0.0265(18)$ & $0.0259(17)$ & $0.0254(15)$ & $0.0063(13)$ & $0.0081(13)$ & $-0.0006(13)$ \\
C7 & $0.031(2)$ & $0.046(2)$ & $0.0276(17)$ & $-0.0068(15)$ & $-0.0032(14)$ & $0.0033(15)$ \\
C8 & $0.0233(17)$ & $0.0218(15)$ & $0.0235(15)$ & $0.0015(12)$ & $0.0098(13)$ & $-0.0025(12)$ \\
C9 & $0.0202(17)$ & $0.0208(15)$ & $0.0249(15)$ & $0.0036(12)$ & $0.0085(12)$ & $-0.0041(12)$ \\
C10 & $0.0204(16)$ & $0.0182(15)$ & $0.0215(14)$ & $0.0006(11)$ & $0.0054(12)$ & $-0.0039(12)$ \\
C11 & $0.0231(16)$ & $0.0179(15)$ & $0.0220(14)$ & $0.0020(11)$ & $0.0046(12)$ & $-0.0017(12)$ \\
C12 & $0.0234(16)$ & $0.0193(15)$ & $0.0221(15)$ & $0.0009(12)$ & $0.0038(12)$ & $0.0056(12)$ \\
C13 & $0.0293(18)$ & $0.0233(16)$ & $0.0295(16)$ & $0.0040(13)$ & $0.0096(13)$ & $0.0055(13)$ \\
C14 & $0.042(2)$ & $0.0283(18)$ & $0.0380(18)$ & $-0.0016(14)$ & $0.0228(16)$ & $0.0014(14)$ \\
C15 & $0.0239(18)$ & $0.0234(16)$ & $0.0246(15)$ & $-0.0028(12)$ & $0.0058(13)$ & $0.0007(13)$ \\
C16 & $0.032(2)$ & $0.0311(19)$ & $0.0285(17)$ & $-0.0004(14)$ & $0.0039(15)$ & $-0.0037(14)$ \\
& & & & & & \\
\hline
\end{tabular}

Geometric parameters $\left(\AA,{ }^{\circ}\right)$

\begin{tabular}{|c|c|c|c|}
\hline $\mathrm{S} 1-\mathrm{O} 2$ & $1.431(2)$ & $\mathrm{C} 5-\mathrm{C} 6$ & $1.385(4)$ \\
\hline $\mathrm{S} 1-\mathrm{O} 1$ & $1.435(2)$ & $\mathrm{C} 5-\mathrm{H} 5$ & 0.9300 \\
\hline $\mathrm{S} 1-\mathrm{N} 1$ & $1.649(2)$ & $\mathrm{C} 6-\mathrm{H} 6$ & 0.9300 \\
\hline $\mathrm{S} 1-\mathrm{C} 1$ & $1.758(3)$ & $\mathrm{C} 7-\mathrm{H} 7 \mathrm{~A}$ & 0.9600 \\
\hline $\mathrm{F} 1-\mathrm{C} 16$ & $1.326(6)$ & $\mathrm{C} 7-\mathrm{H} 7 \mathrm{~B}$ & 0.9600 \\
\hline $\mathrm{F} 2-\mathrm{C} 16$ & $1.311(6)$ & $\mathrm{C} 7-\mathrm{H} 7 \mathrm{C}$ & 0.9600 \\
\hline $\mathrm{F} 3-\mathrm{C} 16$ & $1.323(7)$ & $\mathrm{C} 8-\mathrm{C} 9$ & $1.509(4)$ \\
\hline $\mathrm{F} 1{ }^{\prime}-\mathrm{C} 16$ & $1.302(7)$ & $\mathrm{C} 8-\mathrm{H} 8 \mathrm{~A}$ & 0.9700 \\
\hline $\mathrm{F} 2{ }^{\prime}-\mathrm{C} 16$ & $1.327(7)$ & $\mathrm{C} 8-\mathrm{H} 8 \mathrm{~B}$ & 0.9700 \\
\hline $\mathrm{F} 3^{\prime}-\mathrm{C} 16$ & $1.312(7)$ & C9-H9A & 0.9700 \\
\hline $\mathrm{O} 3-\mathrm{C} 15$ & $1.230(4)$ & C9-H9B & 0.9700 \\
\hline $\mathrm{O} 4-\mathrm{C} 15$ & $1.259(3)$ & $\mathrm{C} 10-\mathrm{C} 11$ & $1.512(4)$ \\
\hline $\mathrm{N} 1-\mathrm{C} 11$ & $1.470(4)$ & $\mathrm{C} 10-\mathrm{H} 10 \mathrm{~A}$ & 0.9700 \\
\hline $\mathrm{N} 1-\mathrm{C} 8$ & $1.478(3)$ & $\mathrm{C} 10-\mathrm{H} 10 \mathrm{~B}$ & 0.9700 \\
\hline $\mathrm{N} 2-\mathrm{C} 9$ & $1.498(3)$ & C11-H11A & 0.9700 \\
\hline $\mathrm{N} 2-\mathrm{C} 10$ & $1.499(3)$ & C11-H11B & 0.9700 \\
\hline $\mathrm{N} 2-\mathrm{C} 12$ & $1.521(3)$ & $\mathrm{C} 12-\mathrm{C} 13$ & $1.514(4)$ \\
\hline $\mathrm{N} 2-\mathrm{H} 2 \mathrm{~A}$ & $0.902(11)$ & $\mathrm{C} 12-\mathrm{C} 14$ & $1.519(4)$ \\
\hline $\mathrm{C} 1-\mathrm{C} 2$ & $1.389(4)$ & $\mathrm{C} 12-\mathrm{H} 12$ & 0.9800 \\
\hline $\mathrm{C} 1-\mathrm{C} 6$ & $1.393(4)$ & $\mathrm{C} 13-\mathrm{H} 13 \mathrm{~A}$ & 0.9600 \\
\hline $\mathrm{C} 2-\mathrm{C} 3$ & $1.382(4)$ & $\mathrm{C} 13-\mathrm{H} 13 \mathrm{~B}$ & 0.9600 \\
\hline $\mathrm{C} 2-\mathrm{H} 2$ & 0.9300 & $\mathrm{C} 13-\mathrm{H} 13 \mathrm{C}$ & 0.9600 \\
\hline $\mathrm{C} 3-\mathrm{C} 4$ & $1.391(4)$ & $\mathrm{C} 14-\mathrm{H} 14 \mathrm{~A}$ & 0.9600 \\
\hline $\mathrm{C} 3-\mathrm{H} 3$ & 0.9300 & $\mathrm{C} 14-\mathrm{H} 14 \mathrm{~B}$ & 0.9600 \\
\hline $\mathrm{C} 4-\mathrm{C} 5$ & $1.386(4)$ & $\mathrm{C} 14-\mathrm{H} 14 \mathrm{C}$ & 0.9600 \\
\hline $\mathrm{C} 4-\mathrm{C} 7$ & $1.503(4)$ & $\mathrm{C} 15-\mathrm{C} 16$ & $1.531(4)$ \\
\hline $\mathrm{O} 2-\mathrm{S} 1-\mathrm{O} 1$ & $119.92(12)$ & $\mathrm{C} 11-\mathrm{C} 10-\mathrm{H} 10 \mathrm{~A}$ & 109.5 \\
\hline $\mathrm{O} 2-\mathrm{S} 1-\mathrm{N} 1$ & $106.25(12)$ & $\mathrm{N} 2-\mathrm{C} 10-\mathrm{H} 10 \mathrm{~B}$ & 109.5 \\
\hline $\mathrm{O} 1-\mathrm{S} 1-\mathrm{N} 1$ & $106.09(11)$ & $\mathrm{C} 11-\mathrm{C} 10-\mathrm{H} 10 \mathrm{~B}$ & 109.5 \\
\hline $\mathrm{O} 2-\mathrm{S} 1-\mathrm{C} 1$ & $108.45(13)$ & $\mathrm{H} 10 \mathrm{~A}-\mathrm{C} 10-\mathrm{H} 10 \mathrm{~B}$ & 108.1 \\
\hline $\mathrm{O} 1-\mathrm{S} 1-\mathrm{C} 1$ & $109.53(12)$ & $\mathrm{N} 1-\mathrm{C} 11-\mathrm{C} 10$ & $109.5(2)$ \\
\hline
\end{tabular}




\begin{tabular}{|c|c|c|c|}
\hline $\mathrm{N} 1-\mathrm{S} 1-\mathrm{C} 1$ & $105.65(12)$ & $\mathrm{N} 1-\mathrm{C} 11-\mathrm{H} 11 \mathrm{~A}$ & 109.8 \\
\hline $\mathrm{C} 11-\mathrm{N} 1-\mathrm{C} 8$ & $110.4(2)$ & $\mathrm{C} 10-\mathrm{C} 11-\mathrm{H} 11 \mathrm{~A}$ & 109.8 \\
\hline $\mathrm{C} 11-\mathrm{N} 1-\mathrm{S} 1$ & $117.15(18)$ & $\mathrm{N} 1-\mathrm{C} 11-\mathrm{H} 11 \mathrm{~B}$ & 109.8 \\
\hline $\mathrm{C} 8-\mathrm{N} 1-\mathrm{S} 1$ & $115.10(17)$ & $\mathrm{C} 10-\mathrm{C} 11-\mathrm{H} 11 \mathrm{~B}$ & 109.8 \\
\hline $\mathrm{C} 9-\mathrm{N} 2-\mathrm{C} 10$ & $109.7(2)$ & $\mathrm{H} 11 \mathrm{~A}-\mathrm{C} 11-\mathrm{H} 11 \mathrm{~B}$ & 108.2 \\
\hline $\mathrm{C} 9-\mathrm{N} 2-\mathrm{C} 12$ & $111.8(2)$ & $\mathrm{C} 13-\mathrm{C} 12-\mathrm{C} 14$ & $110.2(2)$ \\
\hline $\mathrm{C} 10-\mathrm{N} 2-\mathrm{C} 12$ & $112.3(2)$ & $\mathrm{C} 13-\mathrm{C} 12-\mathrm{N} 2$ & $110.2(2)$ \\
\hline $\mathrm{C} 9-\mathrm{N} 2-\mathrm{H} 2 \mathrm{~A}$ & $109.9(19)$ & $\mathrm{C} 14-\mathrm{C} 12-\mathrm{N} 2$ & $110.2(2)$ \\
\hline $\mathrm{C} 10-\mathrm{N} 2-\mathrm{H} 2 \mathrm{~A}$ & $107(2)$ & $\mathrm{C} 13-\mathrm{C} 12-\mathrm{H} 12$ & 108.7 \\
\hline $\mathrm{C} 12-\mathrm{N} 2-\mathrm{H} 2 \mathrm{~A}$ & $106(2)$ & $\mathrm{C} 14-\mathrm{C} 12-\mathrm{H} 12$ & 108.7 \\
\hline $\mathrm{C} 2-\mathrm{C} 1-\mathrm{C} 6$ & $120.7(3)$ & $\mathrm{N} 2-\mathrm{C} 12-\mathrm{H} 12$ & 108.7 \\
\hline $\mathrm{C} 2-\mathrm{C} 1-\mathrm{S} 1$ & $120.4(2)$ & $\mathrm{C} 12-\mathrm{C} 13-\mathrm{H} 13 \mathrm{~A}$ & 109.5 \\
\hline $\mathrm{C} 6-\mathrm{C} 1-\mathrm{S} 1$ & $118.7(2)$ & $\mathrm{C} 12-\mathrm{C} 13-\mathrm{H} 13 \mathrm{~B}$ & 109.5 \\
\hline $\mathrm{C} 3-\mathrm{C} 2-\mathrm{C} 1$ & $119.2(3)$ & $\mathrm{H} 13 \mathrm{~A}-\mathrm{C} 13-\mathrm{H} 13 \mathrm{~B}$ & 109.5 \\
\hline $\mathrm{C} 3-\mathrm{C} 2-\mathrm{H} 2$ & 120.4 & $\mathrm{C} 12-\mathrm{C} 13-\mathrm{H} 13 \mathrm{C}$ & 109.5 \\
\hline $\mathrm{C} 1-\mathrm{C} 2-\mathrm{H} 2$ & 120.4 & $\mathrm{H} 13 \mathrm{~A}-\mathrm{C} 13-\mathrm{H} 13 \mathrm{C}$ & 109.5 \\
\hline $\mathrm{C} 2-\mathrm{C} 3-\mathrm{C} 4$ & $121.0(3)$ & $\mathrm{H} 13 \mathrm{~B}-\mathrm{C} 13-\mathrm{H} 13 \mathrm{C}$ & 109.5 \\
\hline $\mathrm{C} 2-\mathrm{C} 3-\mathrm{H} 3$ & 119.5 & $\mathrm{C} 12-\mathrm{C} 14-\mathrm{H} 14 \mathrm{~A}$ & 109.5 \\
\hline $\mathrm{C} 4-\mathrm{C} 3-\mathrm{H} 3$ & 119.5 & $\mathrm{C} 12-\mathrm{C} 14-\mathrm{H} 14 \mathrm{~B}$ & 109.5 \\
\hline $\mathrm{C} 5-\mathrm{C} 4-\mathrm{C} 3$ & $118.9(3)$ & $\mathrm{H} 14 \mathrm{~A}-\mathrm{C} 14-\mathrm{H} 14 \mathrm{~B}$ & 109.5 \\
\hline $\mathrm{C} 5-\mathrm{C} 4-\mathrm{C} 7$ & $120.6(3)$ & $\mathrm{C} 12-\mathrm{C} 14-\mathrm{H} 14 \mathrm{C}$ & 109.5 \\
\hline $\mathrm{C} 3-\mathrm{C} 4-\mathrm{C} 7$ & $120.5(3)$ & $\mathrm{H} 14 \mathrm{~A}-\mathrm{C} 14-\mathrm{H} 14 \mathrm{C}$ & 109.5 \\
\hline $\mathrm{C} 6-\mathrm{C} 5-\mathrm{C} 4$ & $121.0(3)$ & $\mathrm{H} 14 \mathrm{~B}-\mathrm{C} 14-\mathrm{H} 14 \mathrm{C}$ & 109.5 \\
\hline $\mathrm{C} 6-\mathrm{C} 5-\mathrm{H} 5$ & 119.5 & $\mathrm{O} 3-\mathrm{C} 15-\mathrm{O} 4$ & $128.9(3)$ \\
\hline $\mathrm{C} 4-\mathrm{C} 5-\mathrm{H} 5$ & 119.5 & $\mathrm{O} 3-\mathrm{C} 15-\mathrm{C} 16$ & $116.1(3)$ \\
\hline $\mathrm{C} 5-\mathrm{C} 6-\mathrm{C} 1$ & $119.1(3)$ & $\mathrm{O} 4-\mathrm{C} 15-\mathrm{C} 16$ & $115.0(3)$ \\
\hline $\mathrm{C} 5-\mathrm{C} 6-\mathrm{H} 6$ & 120.5 & $\mathrm{~F} 1{ }^{\prime}-\mathrm{C} 16-\mathrm{F} 2$ & $74.1(6)$ \\
\hline $\mathrm{C} 1-\mathrm{C} 6-\mathrm{H} 6$ & 120.5 & $\mathrm{~F} 1^{\prime}-\mathrm{C} 16-\mathrm{F} 3^{\prime}$ & $106.7(6)$ \\
\hline $\mathrm{C} 4-\mathrm{C} 7-\mathrm{H} 7 \mathrm{~A}$ & 109.5 & $\mathrm{~F} 2-\mathrm{C} 16-\mathrm{F}^{\prime}$ & $123.3(6)$ \\
\hline $\mathrm{C} 4-\mathrm{C} 7-\mathrm{H} 7 \mathrm{~B}$ & 109.5 & $\mathrm{~F} 1{ }^{\prime}-\mathrm{C} 16-\mathrm{F} 3$ & $130.1(6)$ \\
\hline $\mathrm{H} 7 \mathrm{~A}-\mathrm{C} 7-\mathrm{H} 7 \mathrm{~B}$ & 109.5 & $\mathrm{~F} 2-\mathrm{C} 16-\mathrm{F} 3$ & $107.7(6)$ \\
\hline $\mathrm{C} 4-\mathrm{C} 7-\mathrm{H} 7 \mathrm{C}$ & 109.5 & $\mathrm{~F} 3{ }^{\prime}-\mathrm{C} 16-\mathrm{F} 3$ & $29.5(5)$ \\
\hline $\mathrm{H} 7 \mathrm{~A}-\mathrm{C} 7-\mathrm{H} 7 \mathrm{C}$ & 109.5 & $\mathrm{~F} 1{ }^{\prime}-\mathrm{C} 16-\mathrm{F} 1$ & $35.9(5)$ \\
\hline $\mathrm{H} 7 \mathrm{~B}-\mathrm{C} 7-\mathrm{H} 7 \mathrm{C}$ & 109.5 & $\mathrm{~F} 2-\mathrm{C} 16-\mathrm{F} 1$ & $105.3(5)$ \\
\hline $\mathrm{N} 1-\mathrm{C} 8-\mathrm{C} 9$ & $110.5(2)$ & $\mathrm{F} 3{ }^{\prime}-\mathrm{C} 16-\mathrm{F} 1$ & $75.0(6)$ \\
\hline $\mathrm{N} 1-\mathrm{C} 8-\mathrm{H} 8 \mathrm{~A}$ & 109.6 & $\mathrm{~F} 3-\mathrm{C} 16-\mathrm{F} 1$ & $103.4(5)$ \\
\hline $\mathrm{C} 9-\mathrm{C} 8-\mathrm{H} 8 \mathrm{~A}$ & 109.6 & $\mathrm{~F} 1^{\prime}-\mathrm{C} 16-\mathrm{F} 2^{\prime}$ & $108.3(6)$ \\
\hline $\mathrm{N} 1-\mathrm{C} 8-\mathrm{H} 8 \mathrm{~B}$ & 109.6 & $\mathrm{~F} 2-\mathrm{C} 16-\mathrm{F} 2{ }^{\prime}$ & $35.5(5)$ \\
\hline $\mathrm{C} 9-\mathrm{C} 8-\mathrm{H} 8 \mathrm{~B}$ & 109.6 & $\mathrm{~F} 3^{\prime}-\mathrm{C} 16-\mathrm{F} 2^{\prime}$ & $103.5(7)$ \\
\hline $\mathrm{H} 8 \mathrm{~A}-\mathrm{C} 8-\mathrm{H} 8 \mathrm{~B}$ & 108.1 & $\mathrm{~F} 3-\mathrm{C} 16-\mathrm{F} 2{ }^{\prime}$ & $78.5(6)$ \\
\hline $\mathrm{N} 2-\mathrm{C} 9-\mathrm{C} 8$ & $111.8(2)$ & $\mathrm{F} 1-\mathrm{C} 16-\mathrm{F} 2^{\prime}$ & $132.7(6)$ \\
\hline $\mathrm{N} 2-\mathrm{C} 9-\mathrm{H} 9 \mathrm{~A}$ & 109.3 & $\mathrm{~F} 1{ }^{\prime}-\mathrm{C} 16-\mathrm{C} 15$ & $111.1(5)$ \\
\hline $\mathrm{C} 8-\mathrm{C} 9-\mathrm{H} 9 \mathrm{~A}$ & 109.3 & $\mathrm{~F} 2-\mathrm{C} 16-\mathrm{C} 15$ & $115.6(4)$ \\
\hline $\mathrm{N} 2-\mathrm{C} 9-\mathrm{H} 9 \mathrm{~B}$ & 109.3 & $\mathrm{~F} 3{ }^{\prime}-\mathrm{C} 16-\mathrm{C} 15$ & $116.1(5)$ \\
\hline $\mathrm{C} 8-\mathrm{C} 9-\mathrm{H} 9 \mathrm{~B}$ & 109.3 & $\mathrm{~F} 3-\mathrm{C} 16-\mathrm{C} 15$ & $112.0(5)$ \\
\hline $\mathrm{H} 9 \mathrm{~A}-\mathrm{C} 9-\mathrm{H} 9 \mathrm{~B}$ & 107.9 & $\mathrm{~F} 1-\mathrm{C} 16-\mathrm{C} 15$ & $112.0(4)$ \\
\hline $\mathrm{N} 2-\mathrm{C} 10-\mathrm{C} 11$ & $110.8(2)$ & $\mathrm{F} 2{ }^{\prime}-\mathrm{C} 16-\mathrm{C} 15$ & $110.6(5)$ \\
\hline $\mathrm{N} 2-\mathrm{C} 10-\mathrm{H} 10 \mathrm{~A}$ & 109.5 & & \\
\hline
\end{tabular}




$\begin{array}{ll}\mathrm{O} 2-\mathrm{S} 1-\mathrm{N} 1-\mathrm{C} 11 & -172.47(19) \\ \mathrm{O} 1-\mathrm{S} 1-\mathrm{N} 1-\mathrm{C} 11 & -43.8(2) \\ \mathrm{C} 1-\mathrm{S} 1-\mathrm{N} 1-\mathrm{C} 11 & 72.4(2) \\ \mathrm{O} 2-\mathrm{S} 1-\mathrm{N} 1-\mathrm{C} 8 & 55.2(2) \\ \mathrm{O} 1-\mathrm{S} 1-\mathrm{N} 1-\mathrm{C} 8 & -176.13(19) \\ \mathrm{C} 1-\mathrm{S} 1-\mathrm{N} 1-\mathrm{C} 8 & -59.9(2) \\ \mathrm{O} 2-\mathrm{S} 1-\mathrm{C} 1-\mathrm{C} 2 & 163.9(2) \\ \mathrm{O} 1-\mathrm{S} 1-\mathrm{C} 1-\mathrm{C} 2 & 31.4(3) \\ \mathrm{N} 1-\mathrm{S} 1-\mathrm{C} 1-\mathrm{C} 2 & -82.5(2) \\ \mathrm{O} 2-\mathrm{S} 1-\mathrm{C} 1-\mathrm{C} 6 & -20.4(3) \\ \mathrm{O} 1-\mathrm{S} 1-\mathrm{C} 1-\mathrm{C} 6 & -152.9(2) \\ \mathrm{N} 1-\mathrm{S} 1-\mathrm{C} 1-\mathrm{C} 6 & 93.2(2) \\ \mathrm{C} 6-\mathrm{C} 1-\mathrm{C} 2-\mathrm{C} 3 & -1.7(4) \\ \mathrm{S} 1-\mathrm{C} 1-\mathrm{C} 2-\mathrm{C} 3 & 173.9(2) \\ \mathrm{C} 1-\mathrm{C} 2-\mathrm{C} 3-\mathrm{C} 4 & -0.6(4) \\ \mathrm{C} 2-\mathrm{C} 3-\mathrm{C} 4-\mathrm{C} 5 & 2.0(4) \\ \mathrm{C} 2-\mathrm{C} 3-\mathrm{C} 4-\mathrm{C} 7 & -177.8(3) \\ \mathrm{C} 3-\mathrm{C} 4-\mathrm{C} 5-\mathrm{C} 6 & -1.1(4) \\ \mathrm{C} 7-\mathrm{C} 4-\mathrm{C} 5-\mathrm{C} 6 & 178.7(3) \\ \mathrm{C} 4-\mathrm{C} 5-\mathrm{C} 6-\mathrm{C} 1 & -1.1(4) \\ \mathrm{C} 2-\mathrm{C} 1-\mathrm{C} 6-\mathrm{C} 5 & 2.5(4) \\ \mathrm{S} 1-\mathrm{C} 1-\mathrm{C} 6-\mathrm{C} 5 & -173.2(2) \\ \mathrm{C} 11-\mathrm{N} 1-\mathrm{C} 8-\mathrm{C} 9 & 58.7(3) \\ \mathrm{S} 1-\mathrm{N} 1-\mathrm{C} 8-\mathrm{C} 9 & -165.91(18) \\ \end{array}$

$54.2(3)$

$179.4(2)$

$-55.7(3)$

$-56.1(3)$

178.9 (2)

$-60.6(3)$

164.97 (18)

$59.7(3)$

$57.2(3)$

$-179.0(2)$

$179.1(2)$

$-57.1(3)$

$67.0(11)$

$-112.9(10)$

$-14.9(9)$

$165.2(9)$

$-170.9(9)$

$9.3(10)$

$-138.8(8)$

$41.4(8)$

$105.7(9)$

$-74.2(9)$

$-53.3(11)$

$126.8(10)$

Hydrogen-bond geometry $\left(\AA,{ }^{\circ}\right)$

\begin{tabular}{lllll}
\hline$D-\mathrm{H} \cdots A$ & $D-\mathrm{H}$ & $\mathrm{H} \cdots A$ & $D \cdots A$ & $D-\mathrm{H} \cdots A$ \\
\hline $\mathrm{N} 2-\mathrm{H} 2 A \cdots \mathrm{O} 4^{\mathrm{i}}$ & $0.90(1)$ & $1.83(1)$ & $2.724(3)$ & $172(3)$ \\
$\mathrm{C} 9-\mathrm{H} 9 A \cdots \mathrm{O} 2^{\mathrm{ii}}$ & 0.97 & 2.52 & $3.405(4)$ & 151 \\
$\mathrm{C} 10-\mathrm{H} 10 A \cdots \mathrm{O} 4^{\mathrm{ii}}$ & 0.97 & 2.45 & $3.357(4)$ & 155 \\
$\mathrm{C} 2-\mathrm{H} 2 \cdots \mathrm{O}^{\mathrm{iii}}$ & 0.93 & 2.58 & $3.225(4)$ & 127 \\
\hline
\end{tabular}

Symmetry codes: (i) $x+1, y, z$; (ii) $-x+1,-y,-z$; (iii) $x+1, y-1, z$. 